\title{
Effect of Dehydroepiandrosterone Sulfate on the Modulation of Memory and Learning in Sprague-Dawley Rat Hippocampus
}

\author{
Asma Ulhusna Shaimi ${ }^{1}$, Hasmah Abdullah ${ }^{1}$, Zalina Ismail ${ }^{2}$, Wan Amir Nizam Wan Ahmad ${ }^{1}$ \\ ${ }^{1}$ School of Health Sciences, Universiti Sains Malaysia, 16150 Kubang Kerian, Kelantan \\ ${ }^{2}$ Research and Creativity Management Office, Universiti Sains Malaysia, 11800 Penang \\ ${ }^{2}$ Email: drzalina@gmail.com
}

\begin{abstract}
Dehydroepiandrosterone sulphate (DHEAS) is a neurosteroid that is found in greater concentration within the brain rather than in any other body organ (Corpechot et al., 1981) and studies have shown that in the brain, DHEAS has a role in enhancing both learning and memory (Markowski et.al., 2001). This present study investigated the relationship between DHEAS and spatial learning and memory in the rat hippocampus. Male Sprague-Dawley rats were divided into two groups and their spatial learning behaviour was evaluated with the Morris Water Maze. The intensity of DHEAS was simultaneously recorded in real time via the Fiber Fluorescence Microscopy (FFM) S-650 probe of the Cellvisio system. There were significant changes in the swimming pattern of the experimental groups obtained via the Morris Water Maze from day 1 until day 5 and day 6 for the probe test. Meanwhile, it was also seen that the intensity of DHEAS fluorescence increased in parallel to the swimming pattern of the experimental rats in comparison to the control group. The findings suggest that the changes in DHEAS fluorescence has a strong link to both spatial learning and memory.
\end{abstract}

Keywords: DHEAS, Imaging, Learning and memory, Morris water maze

\section{INTRODUCTION}

Baulieu (1991) was the first demonstrate neurosteroids that are synthesized de novo in the rat brain. Dehydroepiandrosterone sulfate (DHEAS) is one of vital neurosteroids, which decreases with age (Sorwell \& Urbanski 2010). Previous studies have been shown that DHEAS declined during brain aging in humans, animals, and cellular model; paralleled by a loss of memory (Racchi et al., 2003; Caruso et al. 2013; Grimm \& Eckert 2017). The result showed that the concentration of DHEAS was higher in the brain than in the plasma (Corpèchot et al. 1981; Dong \& Zheng 2011). However, due to low levels of steroidogenic proteins in the brain and lack of sensitivity of the existing technique, neurosteroidogenesis has not been well elucidated (Kawato et al. 2003).

DHEAS act as modulator of N-methyl-d-aspartate (NMDA) receptors in the brain. The finding reported that the enzymes P450scc and P450c17 for conversion neurosteroids from cholesterol were stimulate of NMDA receptor (Kimoto et al. 2001; Maninger et al. 2009). Several studies suggest that DHEAS can be effective in memory and learning enhancement in animals as well in humans. DHEAS levels correlate with cognitive impairments such as Alzheimer's disease and characterized by age and stress-related cognitive (Corrêa et al. 2016). Some studies showed that DHEAS as a neuroprotective against of cognitive disorders such as Alzheimer's disease (Aly et al. 2011). These results suggested DHEAS play a therapeutic role in the cognitive impairments such as Alzheimer's disease.

DHEAS have been reported that effect memory enhancement in humans and rodents. First reported that
DHEAS significantly enhanced retention of footshock active avoidance training (Flood et al. 1988). In animal models, the performance significantly decreased of latencies and distances. The findings may prove to be relevant a role of DHEAS in memory enhancement. Administration of DHEAS showed that aged animals took longer time to reach the platform but the finding did not improve spatial cognition either young or aged animals (Bodensteiner et al. 2008). A review of the literature found a positive correlation of relationship between DHEAS and global cognition in women and men, and positive correlations with working memory, attention and verbal fluency in women only. These findings demonstrate that DHEAS may affect the cognitive function (De Menezes et al. 2016).

Specifically, we have investigated the effects of DHEAS on the modulation of learning and memory in Sprague-Dawley rat hippocampus.

\section{MATERIALS AND METHODS}

\section{Subjects}

Adult male Sprague-Dawley rats of 8 weeks of age and weighing $250-280 \mathrm{~g}$ were procured from Animal Research and Service Centre (ARASC), Universiti Sains Malaysia, Kelantan, Malaysia. They were maintained under controlled conditions $\left(27 \pm 2^{\circ} \mathrm{C}\right.$ temperature; $40 \pm 5 \%$ relative humidity). Animals were given standard rat pelleted feed and water ad libitum with a $12 \mathrm{~h}$ light-dark cycle with lights going on at 08 00h. The experimental protocols were approved by the Institutional Animal Ethics Committee. 


\section{Surgery}

For implantation guide cannula, animals were anaesthetised with isoflurane gas (3-5\% to induce, $1.5 \%$ maintenance) and positioned in stereotaxis frame. A midline skull incision was made. Using motorised stereotaxis, guide cannula was implanted into the CA1 region of the hippocampus (anterioposterior, $-3.00 \mathrm{~mm}$; mediolateral, $1.0 \mathrm{~mm}$; dorsoventral, $1.25 \mathrm{~mm}$ from bregma) (Paxinos \& Watson 2004). Guide cannula were permanently mounted to the skull with three screws and dental cement. Animals were allowed one week to recover from surgery.

\section{Fluorescence Labeling}

After recovery from surgery the day of behavioural task, freely moving rats were injected with DHEASAlexa Fluor 488 dyes. Injection needles were connected with polyethylene tubing to a microsyringe $(10 \mu \mathrm{L})$ driven by the infusion pump of motorised stereotaxis (brand). Solutions were infused during 1 min at a constant rate of $0.5 \mu \mathrm{L} / \mathrm{min}$. Control rats received the same volume $(0.5 \mu \mathrm{L})$ of the same dye at the same rate of infusion. Injection needles were removed from the guide cannula $10 \mathrm{~min}$ after infusions in order to prevent drug reflux.

\section{Morris Water Maze (MWM) Task}

Spatial learning and memory function in rats was assessed in the MWM task by conducting hidden platform acquisition and probe test (Vorhees \& Williams 2006; Ashraf \& Ismail 2015). Rats were placed in the water at point A. On each trial, if rats did not find the platform within $60 \mathrm{~s}$, the experimenter guided them to it. Rats remained on the platform for 10 $\mathrm{s}$ to allow orientation to visual cues. Rats were allowed to rest for 2 min between trials. Rats were tested in four trials a day for 5 consecutive days. After training acquisition, a probe test was conducted by removing the platform. The rats were allowed to swim and recorded the percentage of time that they spent in the target quadrant and non-target quadrant.

\section{Imaging with Fiber Fluorescence Microscopy (FFM)}

The S-650 FFM probe with a holder was placed into the mount of the stereotaxis device. The probe was fixed on top of the implantation and set the bregma point. Fluorescence labeling were injected before and after behavioral task. The expression of DHEAS were calculated of the intensity of DHEAS in the CA1 region of the hippocampus. The FFM probe was connected to Cell visio Lab system, and the excitation and emission of cells were measured at $488 \mathrm{~nm}$ and 594 $\mathrm{nm}$, respectively.

\section{Statistical Analysis}

Data were represented as mean \pm SEM. Analyses involving two data sets were performed with a two-way ANOVA for Morris water maze task and analysis paired t-test for quantifying the DHEAS of modulation learning and memory. Significance was set as a $p$-value of less than 0.05 . We performed statistical analysis using graph pad Prism software.

\section{RESULTS AND DISCUSSION}

During training, learning process occurred by the measurement of escape latency by time elapsed the rats found the platform. Figure 1 illustrates the swimming pattern during training indicating spatial learning occurred.

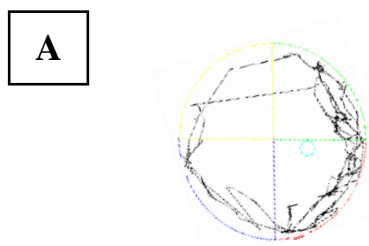

D1

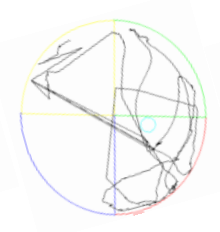

D2

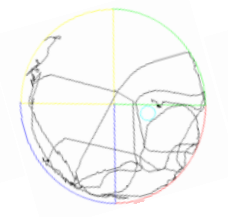

D3

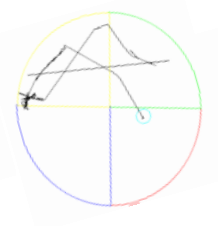

D4

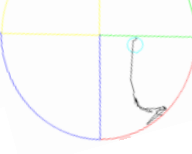

D5

Figure 1. Representative swimming pattern to find the hidden platform from day 1 until day 5. The diagram shows that the training become more easily found the platform through repeated trials each day.
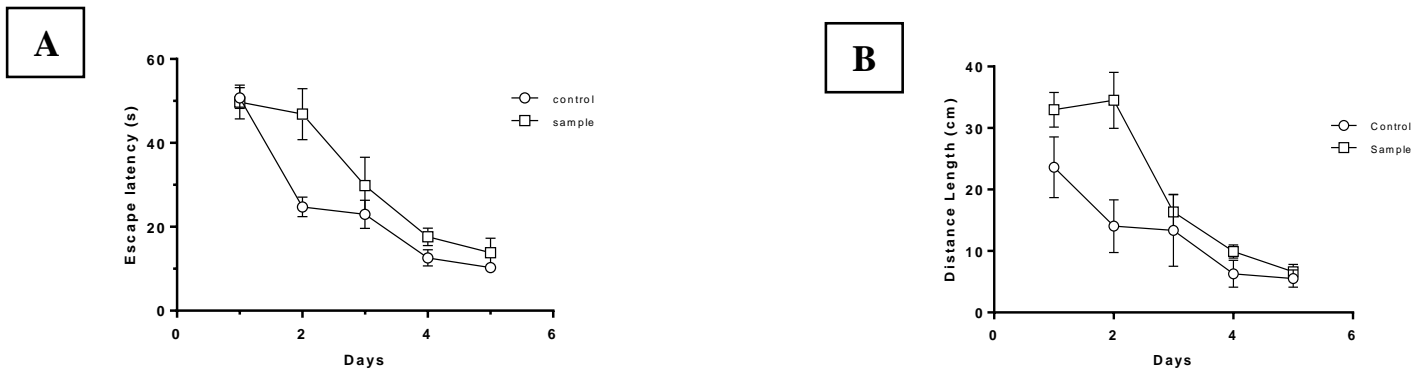

Figure 2. A) Escape latency, and B) Distance length during the training days. Data are presented as the mean \pm SEM ( $\mathrm{n}=8$ animals). The graph shows statistical significance, $p<0.05$ (A) and (B) as determined using two-way ANOVA with Greenhouse-Geisser correction for LSD post-hoc tests. 
Escape latency decreased over the 5 days training period to reach the platform (Figure 2). Analysis twoway ANOVA identified a significant reduction in escape latency over training days (main effect of day, $F$ $(4,60)=34.32 p<0.0001)$. There was a significant interaction effect between days and latencies to reach the platform (interaction effect: $F(4,60)=2.694 p=$ $0.0392)$. For (B), the animals also had significantly decreased of distance length $(F(4,60)=37.21 p<$ $0.0001)$ comparison to control. There was a significant interaction effect between days and distance length to reach the platform (interaction effect: $F(4,60)=6.036$ $p=0.0004)$.

\section{A}
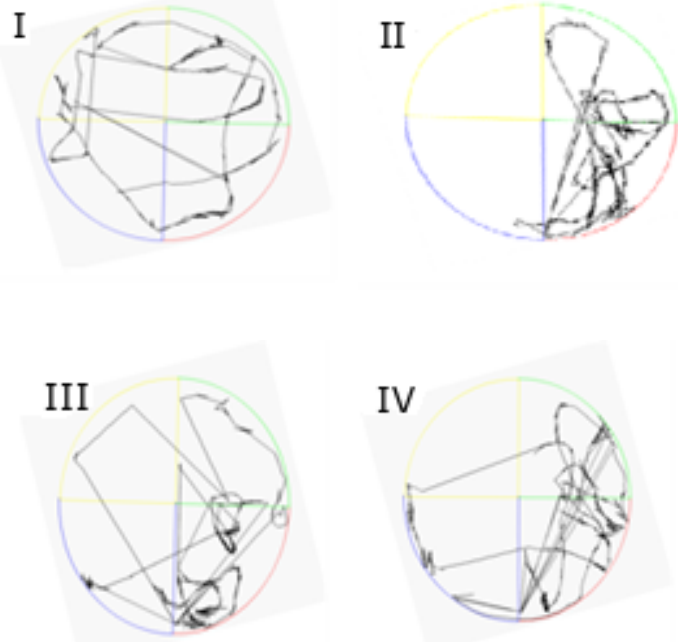

\section{B}

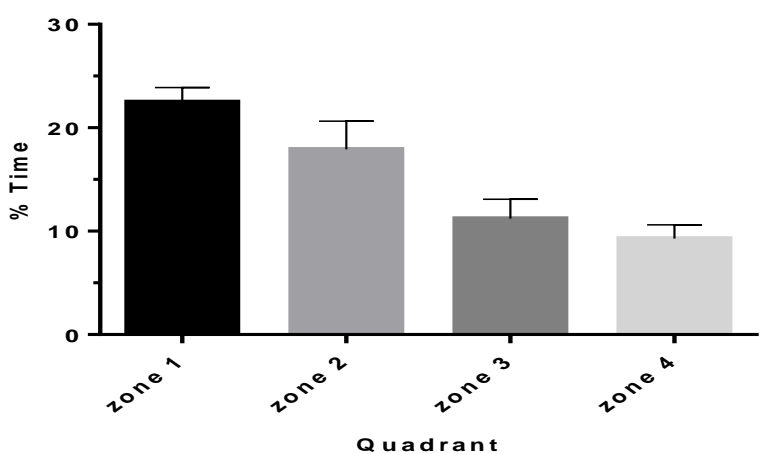

Figure 3. Probe test administered as an assessment of memory on day 6 . A) The swimming pattern while doing the probe test with four trials, B) The graph shows the target quadrant (zone 1) relative to non-target quadrant (zone 2, 3 and 4). Data are presented as the mean \pm SEM $(n=8$ animals). Significant different from target quadrant at $p<0.05$ as determined by two-way ANOVA with the Greenhouse-Geisser correction for LSD post hoc tests.

The probe test was administered on day 6 to assess memory. Data were normally distributed. Analysis two- way ANOVA revealed that a significantly increased percentage of time $F(3,48)=23.28 p<0.0001)$ in the target quadrant (zone 1) relative to the non-target quadrants (zone 2, 3, and 4). There was a significant of interaction effect $(F(3,48)=3.535 p=0.0215)$. The means of sample in zone $1(22.49 \% \pm 1.6)$ spending more time than the other zone.

\section{A}

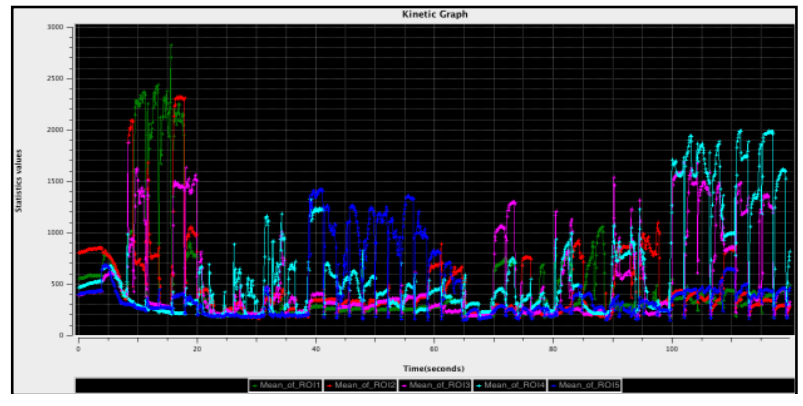

\section{B}
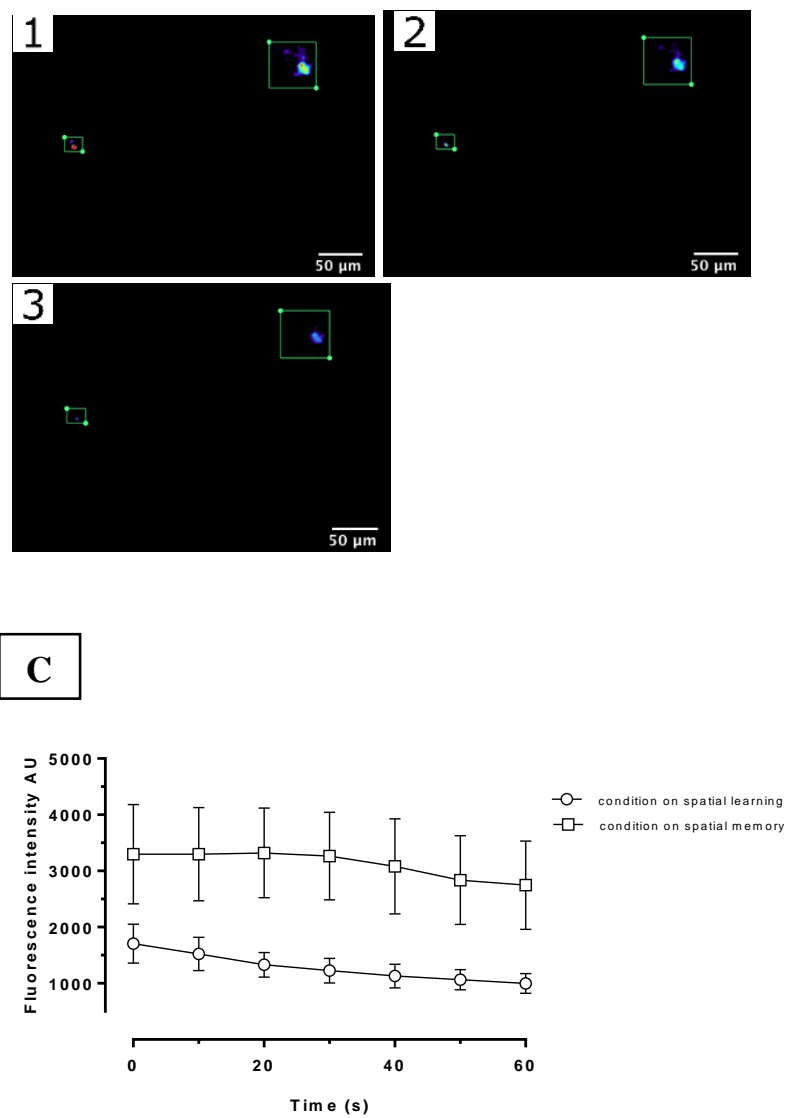

Figure 4. Fibered fluorescence microscopy visualise and evaluate the changes of DHEAS level in the CA1 hippocampus. A) The kinetic graph indicates the region of interest of DHEAS signal after behavioural task. B) The expression of DHEAS fluorescence over time, and C) The graph shows the condition on spatial learning and spatial memory. Data are presented as the mean \pm SEM ( $n=8$ animals). Significant different at $p<$ 0.05 as determined by paired $t$-test with LSD post hoc $t$-test. 
To examine the effect of DHEAS on the modulation of learning and memory, we quantified the changes of DHEAS levels occurred during training and probe test. Data were normally distributed. Analysis paired $t$-test were performed ( $\mathrm{df}=6, t=36.07, \mathrm{p}<0.0001)$ and found the correlation between two group $(\mathrm{r}=0.63, p<$ $0.05)$.

\section{Discussion}

The result showed that swimming pattern had significantly decreased in escape latency and distances. The performance of Sprague-Dawley rats in the Morris water maze exhibited significant changes in spatial learning. Mean latencies to find the hidden platform indicating that all animals have learned the location of the platform over 5 days training. Similarly, the finding of rats required less time to find the platform and increased the time and path length in the target quadrant (Galea et al. 2000; D'Hooge \& De Deyn 2001; Barnhart et al. 2015). Recently reported that demonstrated consistent decreased the escape latency over a 5 days training period (Frye \& Lacey 1999). A few factors when evaluating and comparing the effectiveness of DHEAS on learning and memory are time, dosage, route of administration, and testing paradigm (Bodensteiner et al. 2008). Researchers have reported that no effect on the Morris water maze performance but has effects on the $\mathrm{Y}$ maze performance with injection (s.c.) of DHEAS into ovariectomized rats (Frye \& Sturgis, 1995).

Sample that we did the behavioural task during training days took a longer time and distance length on day 1 until day 3 . Then, the animals were made learning process and become faster to reach the platform on training day 4 and day 5. This finding suggest that the animals having stress and hard to learn on day 1 until day 3 . The implantation of the skull and the surgery process may influence learning process. Some studies have shown DHEAS level associated with depressed mood and anxiety disorders. Association was independent of age, lifestyle, and weight change (Walton et al. 1995; Elizabeth et al., 2015).

Data was collected during the probe test and significantly increased the percentage of time in the target quadrant by given meaning of time within 1 minute. The rats have learned the location of platform, and tried to recognise and remember the location. Therefore, the rats swim and spend more time in the target quadrant (Yin et al. 2013). Similar findings, the weanling mice had significantly increased the percentage of time at the target quadrant (Barnhart et al. 2015). Some studies observed that significantly increased the performance in working memory (Aaseb $\varnothing$ et al. 2018). This study suggested memory enhancement in rats (Galea et al. 1999; Aasebø et al. 2018).

By using FFM probe connected to Cell visio Lab System, the changes of DHEA level can be imaged, recorded and quantified. Several studies have previously investigated this approach enable high speed, high resolution, minimal invasiveness while insert the probe, and allowed the researchers to monitor the signal of fluorescence in real-time imaging (Davenne et al. 2005; Vincent et al. 2006; Chen et al. 2015). The result of the present investigation are consistent increases in probe test over the time of the DHEAS fluorescence intensity in parallel to the swimming pattern of the experimental rats indicated that the changes of DHEAS level in the rat hippocampus obtained. In this study, the evidence that DHEAS effect the modulation of spatial learning and memory enhancement during behavioural test. Researchers have been reported that fluorescence increase in the region of interest while adding bicuculline in response to calcium activity (Vincent et al. 2006). On the other hand, GCaMP3 to detect the calcium signals in dopamine neuron demonstrated that the changes of fluorescence intensity (Gore et al. 2016).

Collectively, the correlation of DHEAS level in the rat hippocampus with the behavioural task for learning and memory can be determined. This finding is the first report of DHEAS fluorescence in the rat hippocampus due to modulation of learning and memory. In addition, the hippocampus, an important location in learning and memory and cognitive impairments in Alzheimer's disease. Cell visio can be one of the new insights to understanding the role of DHEAS in improving learning and memory impairments associated with diseases such as Alzheimer's disease.

\section{CONCLUSIONS}

The intensity of DHEAS was simultaneously recorded in real time via the Fiber Fluorescence Microscopy (FFM) S-650 probe of the Cell visio system. There were significant changes in the swimming pattern of the experimental groups obtained via the Morris Water Maze from day 1 until day 5 and day 6 for the probe test. Meanwhile, it was also seen that the intensity of DHEAS fluorescence increased in parallel to the swimming pattern of the experimental rats in comparison to the control group. The findings suggest that the changes in DHEAS fluorescence has a strong link to both spatial learning and memory. Cell visio can be one of the new insights to understanding the role of DHEAS in improving learning and memory impairments associated with diseases such as Alzheimer's disease

\section{ACKNOWLEDGEMENTS}

This study was supported by Fundamental Research Grant Scheme; FRGS (203/PPSK/6171153) and P3Neuro (304/PPSK/652204/K134). 


\section{REFERENCES}

Aasebø IEJ, Kasture AS, Passseggeri M, Tashiro A. 2018. A behavioural task with more opportunities for memory acquisition promotes the survival of new neurons in the adult dentate gyrus. Scientific Report 8: 7369-7380.

Aly HF, Metwally FM, Ahmed HH. 2011. Neuroprotective effects of dehydroepiandrosterone (DHEA) in rat model of Alzheimer's disease. Acta Biochimica Polonica 58: 513-520.

Ashraf S, Ismail Z. 2015. Chronic Sodium Arsenite Exposure Effects Cognitive Behaviour of Sprague Dawley Rats. American Journal of Materials Science. 5: 21-24.

Barnhart CD, Yang D, Lein PJ. 2015. Using the Morris Water Maze to Assess Spatial Learning and Memory in Weanling Mice. Plos One 10: 1-16.

Baulieu EE. 1991. Neurosteroids: a new function in the brain. Biology of the Cell 71: 3-10.

Bodensteiner KJ, Stone IJ, Ghiraldi LL. 2008. Effects of Dehydroepiandrosterone Sulfate and Progesterone on Spatial Learning and Memory in Young and Aged Mice. The Journal of General Psychology 135: 271-286.

Caruso D, Barron AM, Brown MA, Abbiati F, Carrero P, Pike CJ, Garcia-Segura LM, Melcangi RC. 2013. Age-related changes in neuroactive steroid levels in $3 \times \mathrm{Tg}-\mathrm{AD}$ mice. Neurobiology of Aging 34: 1080-1089.

Chen X, Cao H, Saraf A, Zweifel LS, Storm DR. 2015. Overexpression of the Type 1 Adenylyl Cyclase in the Forebrain Leads to Deficits of Behavioral Inhibition. The Journal of Neuroscience 35: 339-351.

Corpéchot C, Robel P, Axelson M, Sjövall J, Baulieu E. 1981. Characterization and measurement of dehydroepiandrosterone sulfate in rat brain. Proceedings of the National Academy of Sciences 78: 4704-4707

Corrêa MS, Giacobbo BL, Vedovelli K, de Lima DB, Ferrari P, Argimon IIL, Waiz JC, Bromberg E. 2016. Age Effects on Cognitive and Physiological Parameters in Familial Caregivers of Alzheimer's Disease Patients. Plos One 11: 1-16.

Davenne M, Custody C, Charneau P, Lledo P. 2005. In Vivo Imaging of Migrating Neurons in the Mammalian Forebrain. Chemical Senses 30: 115-116.

D'Hooge R, De Deyn PP. 2001. Applications of the Morris water maze in the study of learning and memory. Brain Research Reviews 36: 60-90.

De Menezes KJ, Peixoto C, Nardi AE, Carta MG, Machado S, Veras AB. 2016. Dehydroepiandrosterone, Its Sulfate and Cognitive Functions. Clinical Practice \& Epidemiology in Mental Health 12: 24-37.

Dong Y, Zheng P. 2011. Dehydroepiandrosterone Sulphate: Action and Mechanism in the Brain. Journal of Neuroendocrinology 24: 215-224.

Elizabeth BMD, Denise MMD, Gail ALBS, Adrianna K. 2015. Endogenous Levels of Dehydroepiandrosterone Sulfate, but Not Other Sex Hormones, Are Associated with Depressed Mood in Older Women: The Rancho Bernando Study. Journal of the American Geriatrics Society 47: 685-691.

Flood JF, Smith GE, Roberts E. 1988. Dehydroepiandrosterone and its sulfate enhance memory retention in mice. Brain Research 447: $269-278$
Frye CA, Lacey EH. 1999. The Neurosteroids DHEA and DHEAS May Influence Cognitive Performance by Altering Affective State. Physiology \& Behavior 66: 85-92.

Frye CA, Sturgis JD. 1995. Neurosteroids affect spatial/reference, working, and long-term memory of female rats. Neurobiology of Learning and Memory 64: 83-96.

Galea LAM, Ormerod BK, Sampath S, Kostaras X, Wilkie DM, Phelps MT. 2000. Spatial Working Memory and Hippocampal Size across Pregnancy in Rats. Hormones and Behavior 37: 8695.

Grimm A, Eckert A. 2017. Brain aging and neurodegeneration: from a mitochondrial point of view. Journal of Neurochemistry. 143 418-431.

Gore BB, Soden ME, Zweifel LS. 2016. Visualization of plasticity in fear-evoked calcium signals in midbrain dopamine neurons. Brief Communication 21: 575-579.

Kawato S, Yamada M, Kimoto T. 2003. Brain neurosteroids are $4^{\text {th }}$ generation neuromessengers in the brain: Cell biophysical analysis of steroid signal transduction. Advances in Biophysics 37: $1-48$.

Kimoto T, Tsurugizawa T, Ohta Y, Makino J, Tamura H, Hojo Y, Takata N, Kawato S. 2001. Neurosteroid synthesis by cytochrome p450-containing systems localized in the rat brain hippocampal neurons:N-methyl-D-aspartate and calciumdependent synthesis. Endocrinology 142: 3578-3589.

Maninger N, Wolkowitz OM, Reus VI, Epel ES, Mellon SH. 2009. Neurobiological and Neuropsychiatrict Effects of Dehydroepiandrosterone (DHEA) and DHEA Sulfate (DHEAS). Frontiers in Neuroendocrinology 30: 65-91.

Paxinos G, Watson C. 2004. The Rat Brain in Stereotaxic Coordinates - The New Coronal Set $5^{\text {th }}$ Edition. Australia.

Racchi M, Balduzzi C, Corsini E. 2003. Dehydroepiandrosterone (DHEA) and the Aging Brain: Flipping a Coin in the "Fountain of Youth". CNS Drug Reviews 9: 21-40.

Sorwell KG, Urbanski HF. 2010. Dehydroepiandrosterone and agerelated cognitive decline. Age 32: 61-67.

Vincent P, Maskos U, Charvet I, Bourgeais L, Stoppini L, Leresche N, Changeux J, Lambert R, Meda P, Paupardin-Tritsch D. 2006. Live imaging of neural structure and function by fibred fluorescence microscopy. European Molecular Biology Organization 7: 1154-1161.

Vorhees CV, Williams MT. 2006. Morris water maze: procedures for assessing spatial and related forms of learning and memory. Natural Protocols 1: 848-858.

Walton KG, Pugh ND, Gelderloos P, Macrae P. 1995. Stress reduction and preventing hypertension: Preliminary support for a psychoneuroendocrine mechanisms. Journal of Alternative and Complementary Medicine 3: 263-283.

Wong SY, Leung JC, Kwok T, Ohlsson C, Vandenput L, Leung PC. 2011. Low DHEAS levels are associated with depressive symptoms in elderly Chinese men: results from a large study. Asian Journal of Andrology 13: 898-902.

Yin MM, Wang W, Sun J, Liu S, Liu XL, Niu YM, Yuan HR, Yang FY, Fu L. 2013. Paternal treadmill exercise enhances spatial learning and memory related to hippocampus among male offspring. Behavioural Brain Research 253: 297-304. 
THIS PAGE INTENTIONALLY LEFT BLANK 